\title{
Flavonoids in prevention of diseases with respect to modulation of Ca-pump function
}

\section{Ĺubica HORÁKOVÁ}

Institute of Experimental Pharmacology \& Toxicology, Slovak Academy of Sciences, SK-84104 Bratislava, Slovakia

ITX040311R02 • Received: 14 July 2011 • Revised: 10 August 2011 • Accepted: 13 August 2011

\begin{abstract}
Flavonoids, natural phenolic compounds, are known as agents with strong antioxidant properties. In many diseases associated with oxidative/nitrosative stress and aging they provide multiple biological health benefits. Ca2+-ATPases belong to the main calcium regulating proteins involved in the balance of calcium homeostasis, which is impaired in oxidative/nitrosative stress and related diseases or aging. The mechanisms of Ca2+-ATPases dysfunction are discussed, focusing on cystein oxidation and tyrosine nitration. Flavonoids act not only as antioxidants but are also able to bind directly to Ca2+-ATPases, thus changing their conformation, which results in modulation of enzyme activity.

Dysfunction of $\mathrm{Ca}^{2+}$-ATPases is summarized with respect to their posttranslational and conformational changes in diseases related to oxidative/nitrosative stress and aging. Ca2+-ATPases are discussed as a therapeutic tool and the possible role of flavonoids in this process is suggested.
\end{abstract}

KEY WORDS: flavonoids; oxidative/nitrosative stress; Ca2+-ATPases in aging and diseases

\section{Introduction}

Free oxygen and nitrogen reactive species (ROS, RNS) are participating in aging and age related diseases. The search for mechanisms of oxidative/nitrosative injury involved in diseases and aging is important for finding new therapeutic approaches. Impairment of calcium homeostasis is a consequence of oxidative/nitrosative stress. In recent years, many results were published showing that dysfunction of $\mathrm{Ca}^{2+}$-ATPases, the main calcium level regulating proteins, is included in the above mentioned conditions. In addition, modulation of $\mathrm{Ca}^{2+}$-ATPases by antioxidants may be a therapeutic tool for improvement of complications associated with human diseases and their experimental animal models. Flavonoids, in addition to their antioxidant properties exert beneficial effects based on their ability to bind to $\mathrm{Ca}^{2+}$-ATPases and thus to change their activity.

Correspondence address:

\section{Ĺubica Horáková, PhD.}

Department of Biochemical Pharmacology, Institute of Experimental Pharmacology \& Toxicology, Slovak Academy of Sciences

Dúbravská cesta 9, 84104 Bratislava, Slovak Republic TEL.: +421-2-59410656 • E-MAIL: lubica.horakova@savba.sk
The importance of $\mathrm{Ca}^{2+}$-ATPases, their impairment and impact as a possible therapeutic tool in diseases related to oxidative/nitrosative stress has not yet been summarized. This review deals with the modulation of $\mathrm{Ca}^{2+}$-ATPases by oxidative/nitrosative stress in vitro and in diseases and with their potential therapeutic effect.

Flavonoids are phenolic compounds known as characteristic red, blue and purple anthocyanin pigments of plant tissues (Winkel-Shirley et al., 2001). Flavonoids occur in food either as free monomers (quercetin, catechin) or oligomers (procyanidins), or they are bound to saccharides as glycosides. Consumption of flavonoid-rich food is associated with a lower incidence of coronary heart disease, myocardial infarction, cancer, neurodegenerative psychic diseases, and other chronic diseases. In the pathology of these diseases, oxidative stress has been assumed to play a role and flavonoids have been suggested to exert health benefits through antioxidant mechanisms. In addition to their antioxidant properties, flavonoids have been reported to exhibit other multiple biological effects, antiviral, antibacterial, anti-inflammatory, vasodilatory, anticancer, anti-ischemic, etc. (Procházková et al., 2011). An important aim of the present review is to elucidate another mechanism by which flavonoids may exert health benefits, i.e. the modulation of calcium homeostasis and cell signaling via $\mathrm{Ca}^{2+}$-ATPase. 


\section{Antioxidant properties and structure}

Both the configuration and the total number of hydroxyl groups substancialy influence the mechanism of antioxidant activity (Heim et al., 2002). The basic structure of flavonoids is depicted in Figure 1. The B ring hydroxyl configuration is the most significant determinant of ROS scavenging (Burda \& Oleszek, 2001), whereas substitution of the rings $A$ and $C$ has little influence on superoxide anion radical scavenging (Taubert et al., 2003; Amic et al., 2007). The in vitro antioxidant activity could be increased by polymerization of flavonoid monomers (proanthocyanidins known also as condensed tannins). The polymers of catechins are excellent in vitro antioxidants due to the high number of hydroxyl groups. The glycosylation of flavonoids reduces their in vitro antioxidant activity, compared with corresponding aglycones (Procházková et al., 2011). Glycosylation of the 3-OH group has namely strong suppresive effect on their antioxidant activity (Rice-Evans et al.,1996).

Structural features of flavonoids required for efficient radical scavenging are i) an ortho-dihydroxy (catechol) structure in the B ring for electron delocalization, ii) 2,3-double bond in conjugation with a 4-oxo function in the $\mathrm{C}$ ring providing electron delocalization from the $\mathrm{B}$ ring, iii) hydroxyl groups at positions 3 and 5 providing hydrogen bonding to the oxo group (Croft 2006) (Figure 2).

\section{Ability to activate or inhibit enzymes}

The ability to interact with various antioxidant enzymes belongs also to important mechanisms of flavonoids in preventing injury caused by free radicals. Protective effects of quercetin and catechin against hydrogen peroxide toxicity in cultured rat hepatocytes BL-9 were related to the activation of glutathione peroxidase (GPx) (Nagata et al., 1999). Activation of survival signaling proteins (protein kinase B and extracellular regulated kinases) as well as increase of GPx and glutathione reductase (GR) caused by cocoa flavonoids was observed in human hepatocytes (Martin et al., 2010). Luteonin induced human lung carcinoma cell apoptosis was accompanied by activation of antioxidant enzymes such as superoxide dismutase (SOD) and catalase (CAT) (Leung et al., 2006).

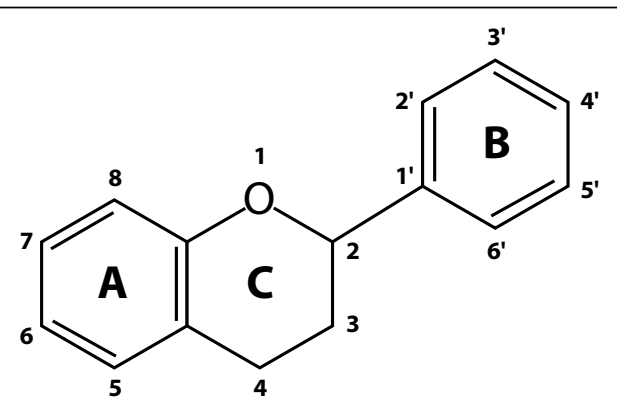

Figure 1. Basic structure of flavonoids.
Flavonoids inhibit the enzymes responsible for superoxide production, e.g. xanthine oxidase and protein kinase C. Flavonoids have been shown to inhibit cyclooxygenase, lipoxygenase, microsomal succinoxidase and NADH oxidase. Flavonoids were found to exert an inhibitory effect on the expression of inducible nitric oxide synthase (NOS), yet they did not inhibit its activity. Several flavonoids, including quercetin, induce a reduction of ischemia-reperfusion injury by interfering with inducible NOS activity (Procházková et al., 2011).

\section{Ability to modulate sarco/endoplasmic reticulum $\mathrm{Ca}^{2+}$-ATPase (SERCA)}

\section{Physiological importance of SERCA}

SERCA is an intracellular membrane-bound enzyme that utilizes the free energy of ATP to transport $\mathrm{Ca}^{2+}$ against a concentration gradient. The physiological role of SERCA is to sequester cytosolic $\mathrm{Ca}^{2+}$ into membranebound intracellular compartments. SERCA-mediated $\mathrm{Ca}^{2+}$ uptake into stores plays a key role in maintenance of intracellular free $\mathrm{Ca}^{2+}$ levels within a physiological range. In addition, during contractions caused by elevated levels of intracellular $\mathrm{Ca}^{2+}$, accelerated sequestration of $\mathrm{Ca}^{2+}$ by SERCA mediates smooth, cardiac, and skeletal muscle relaxation. In non-contractile cells, such as proliferating smooth muscle cells or endothelium, SERCA regulates many cellular processes, including cell growth, apoptosis and migration. $\mathrm{Ca}^{2+}$-ATPase dysfunction has been detected in experimental and human pathology (Tong et al., 2010; Inesi et al., 2005.)

\section{Mechanisms of SERCA regulation}

The mechanisms of intracellular calcium regulation by SERCA, involved in vascular relaxation, in cardiac and muscle relaxation, as well as in growth and differentiation, may include peroxinitrite (generated from nitric oxide and superoxide), which causes glutathione (GSH) to bind to SERCA cysteine thiols (Cohen et al., 2006). GSH, when oxidized, is rapidly reduced and/or resynthetized to protect proteins from oxidation. With RNS GSH forms, S-nitroso glutathione (GSNO), sulfenic acid (GSOH), and GSH thiyl radicals (GS·), which can react with nearby protein thiols. Protein thiolate anions ( ${\mathrm{R}-\mathrm{S}^{-}}^{-}$) are predisposed to react with these species. Chemical adducts of protein thiols generated in the presence of RNS include Pr-SNO (generated by S-nitrosylation), Pr-SSG (generated by glutathiolation) and oxidized thiols $(\mathrm{Pr}-\mathrm{SOH}),\left(\mathrm{Pr}-\mathrm{SO}_{2} \mathrm{H}\right)$, $\left(\mathrm{PrSO}_{3} \mathrm{H}\right)$, as well as intra- and inter-protein dithiols. All modifications, except those of sulfinic acid $\left(\mathrm{Pr}-\mathrm{SO}_{2} \mathrm{H}\right)$ and sulfonic acid $\left(\mathrm{PrSO}_{3} \mathrm{H}\right)$, are reversible under physiologic conditions, enabling them to participate in cell signaling.

Another peroxynitrite product is nitrotyrosine. In SERCA2 in the aging heart and skeletal muscle nitration of vicinal tyrosines, Tyr 294 and Tyr295 was demonstrated on the luminal side of the membrane-spanning helix M4, correlating with partial inhibition of Ca-ATPase activity. This is suggesting a possible regulatory function in 
down-regulating mitochondrial energy production and the associated decrease in generation of reactive oxygen/ nitrogen species (Bigelow, 2007). Nitrotyrosine is generated from nitric-oxide-derived oxidants, mainly of the $\mathrm{CO}_{2}$ adduct of peroxynitrite (ONOO-) or of $\mathrm{NO}_{2}$ (Pacher et al., 2007). Peroxynitrite reacts rapidly with such nucleophiles as protein thiols and transition metal centers, and the physiologically abundant $\mathrm{CO}_{2}$, which occurs in millimolar concentrations in cells. The $\mathrm{CO}_{2}$ adduct nitrosoperoxycarbonate $\left(\mathrm{ONOOCO}_{2}\right)$ rapidly homolyzes to ${ }^{\circ} \mathrm{CO}^{-}{ }_{3}$ (carbonate radical) and $\cdot \mathrm{NO}_{2}$ (nitrogen dioxide) to undergo a radical mechanism of tyrosine nitration. This involves hydrogen atom abstraction from the 3-position of tyrosine to form the tyrosyl radical that rapidly combines with $\cdot \mathrm{NO}_{2}$ forming 3-nitrotyrosine. Protein nitration may also be formed by generation of $\cdot \mathrm{NO}_{2}$ by heme peroxidases, such as myeloperoxidase and eosinophil peroxidase, in the presence of hydrogen peroxide.

Low concentrations of peroxynitrite induce S-glutathiolation of SERCA2 in vivo, whereas higher concentratioins of peroxynitrite result in irreversible modification of Cys ${ }^{674}$ to cystein sulfonic acid $\left(\mathrm{Cys}-\mathrm{SO}_{3} \mathrm{H}\right)$ and accompanying nitrotyrosine modification (Cohen et al., 2006).

\section{SERCA isomers and structure}

Three genes for SERCA have been identified (SERCA1, SERCA2, and SERCA3), and different SERCA isoforms are expressed in tissue-specific and developmentally regulated patterns (Dremina et al., 2007). The fast-twitch muscle isoform SERCA1 is restricted to fast-twich skeletal muscle, while SERCA2a is expressed in cardiac and slow-twitch skeletal muscle. The isoforms SERCA2b and SERCA3a-3f are present in a large variety of tissues, including nonmuscle tissues.

SERCA belongs to the "P" family of cation transport ATPases, all of them forming phosphorylated enzyme intermediates by covalent interaction of the ATP terminal phosphate with an aspartyl residue at the catalytic site (Inesi et al., 2005). ATPase includes a membrane-bound region connected through a stalk to an extramembranous (i.e. cytosolic) headpiece. Ca-binding sites are located within the membrane-bound region, the ATP binding (nucleotide binding) site is situated in the cytosolic region of SERCA (Tupling et al., 2001).

\section{SERCA, oxidation and natural plant products}

SERCA pumps play a central role in maintaining low levels of free cytosolic calcium within cells. This $\mathrm{Ca}^{2+}$ pump is inhibited by a wide spectrum of hydrophobic molecules, a number of which are plant-derived natural products, such as thapsigargin (Wictome et al.,1992), curcumin (Bilmen et al., 2001; Dyer et al., 2002), and the flavonoid quercetin (Shoshan et al., 1981). Flavonoids are able to bind to nucleotide binding sites, thus changing SERCA activity. Therefore one possible mechanism by which apoptosis is initiated by flavonoids is via $\mathrm{Ca}^{2+}$ pump inhibition leading to elevation of cytosolic $\left[\mathrm{Ca}^{2+}\right.$, initiating $\mathrm{Ca}^{2+}$-dependent mitochondrial-mediated cell death (Ogunbayo et al., 2008).

Natural plant extracts with antioxidant properties and prevention against pathological reduction of the Ca-pump may offer dual protection against some cardiovascular, skeletal muscle and inflammatory diseases. Recent findings showed that the inhibitory effect of $\mathrm{HOCl}$, which reduced SR $\mathrm{Ca}^{2+}$-ATPase activity at least partially by structural changes, was prevented by standardized flavonoid extract from leaves of Ginkgo biloba (EGb761) (Štrosová et al., 2005). It is known that flavonoids are able to bind to proteins, thus changing their structure and function (Rohdewald, 2002).

The standardized extracts of plant flavonoids, EGb761 and pycnogenol (Pyc) prevented sulfhydryl (SH) group oxidation and protein carbonyl (PCO) formation. Pyc in addition prevented thiobarbituric acid reactive species (TBARS) formation in SR oxidized by the Fenton system. The lower antioxidant effect of EGb761 compared with Pyc may be associated with the fact that $\mathrm{OH}$-groups of compounds included in this extract and effective in scavenging free radicals are bound to glucose (Drieu,

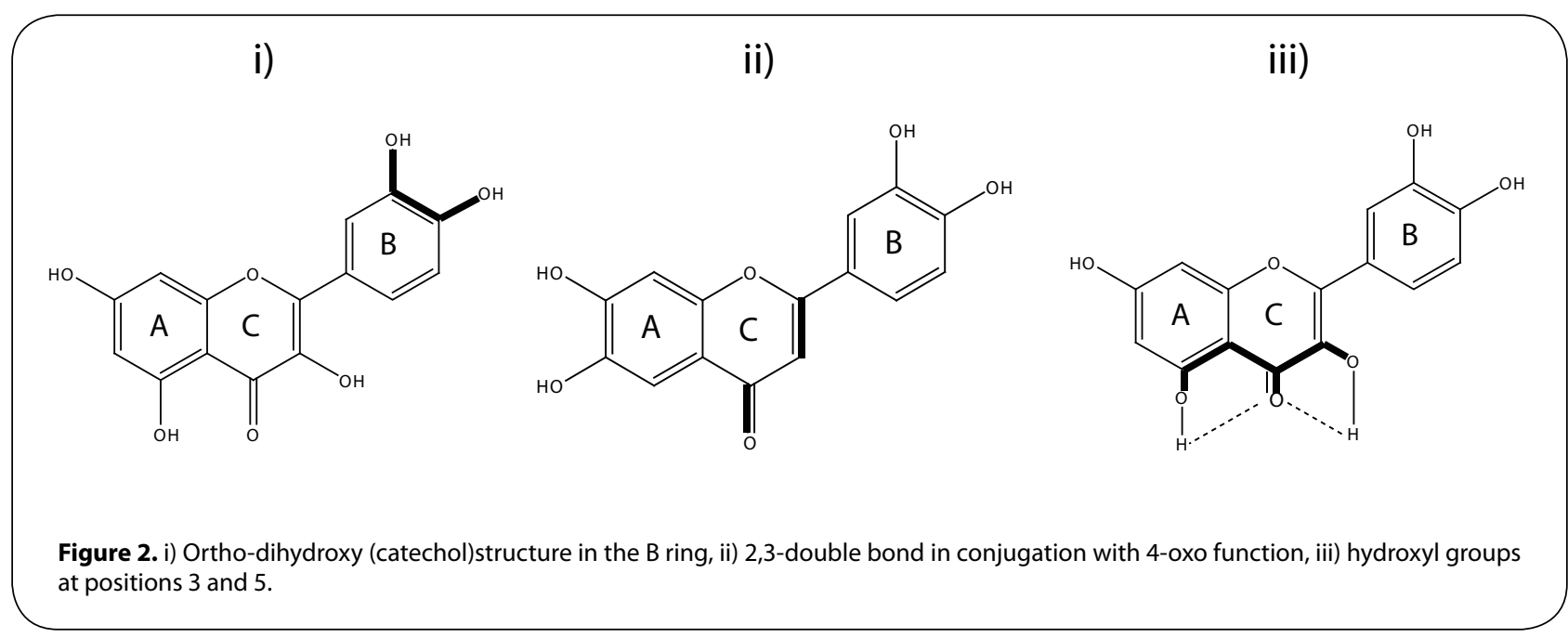


1986). Mixtures of many compounds included in Pyc and EGb761 may have multiple and synergistic effects which could result in effective decrease of protein carbonyl formation. These extracts are also able to chelate Fe-ions and scavenge hydrogen peroxide and hydroxyl-radicals (Rohdewald, 2002; Sastre et al., 1998). In spite of these antioxidant and scavenging effects exerted by EGb761 and Pyc, no protective effects on $\mathrm{Ca}^{2+}$-ATPase activity has been observed, on the contrary, an additional decreasing effect on the activity of this enzyme activity was observed. This effect may be caused by the interaction of flavonoids with free radicals derived from the Fenton system, inducing injury by this secondary reaction. In addition, Pyc is able to inhibit $\mathrm{Ca}^{2+}$-ATPase activity also in the absence of oxidants. It is able to bind to proteins, alter their structure and thereby modulate the activity of key enzymes (Packer et al., 1999). The ability of Pyc to decrease $\mathrm{Ca}^{2+}$-ATPase activity under non-oxidized conditions was reported by Štrosova et al. (2006). Flavonoids included in the above mentioned standardized plant extracts may induce inhibition or stimulation of key enzymes (Rohdewald, 2002). EGb761 or its individual flavonoid compounds are able to bind to protein. Their binding to the nucleotide (ATPbinding) site of $\mathrm{Ca}^{2+}$-ATPase (Schroeter et al., 2000) as well as interaction with ion channels was reported (Ishige et al., 2001). We concluded that modulation of Ca-pump function by antioxidants depended on the mode of oxidative injury (Voss et al., 2008). Both $\mathrm{HOCl}$ and radicals generated by the Fenton reaction are included in the processes of inflammation and are able to induce structural changes in proteins resulting in a decrease of Ca-pump function. The protective effect of trolox and EGb761 on SERCA activity may be based on the interaction of specific ( $\mathrm{HOCl}$ related) secondary oxidative products with antioxidants. On the other hand, the same antioxidants, in spite of the fact that they protected SH groups and prevented TBARS or carbonyl generation, were found to be ineffective in preventing SERCA activity decrease induced by the Fenton reaction associated with $\mathrm{HO}$ and ferryl-radicals generation.

We conclude that the ability to protect $\mathrm{Ca}^{2+}$-ATPase activity by antioxidants depends on the mode of oxidative injury. We further suggest that conformational alterations in cytosolic nucleotide (ATP)-binding or transmembrane $\mathrm{Ca}^{2+-b i n d i n g ~ r e g i o n s ~ o f ~} \mathrm{Ca}^{2+-}$-ATPase, which are now under investigation in our laboratory, may be also critical for protective effects of antioxidants.

\section{Prooxidant properties of flavonoids}

Under certain circumstances, flavonoids can act, as prooxidants, promoting the oxidation of other compounds. The prooxidant activity is probably directly proportional to the total number of hydroxyl groups in the flavonoid molecule (Cao et al., 1997). Multiple hydroxyl groups, especially in the B-ring, significantly increased the production of hydroxyl radicals in the Fenton reaction, while on the other hand, mono- and dihydroflavonoids failed to exert any prooxidant activity (Heim et al., 2002; Hanasaki et al., 1994). There is also evidence that the 2,3-double bond and 4-oxo arrangement of flavones may promote the formation of ROS induced by divalent copper in the presence of oxygen (Sun et al., 2010). Flavonoid prooxidant properties seem to be concentration dependent. An example may be rat liver microsomes, where quercetin and myricetin powerfully inhibited iron-induced lipid peroxidation at low micromolar concentrations (IC50 $\leq 1.5 \mu \mathrm{M})$. However at $100 \mu \mathrm{M}$ concentration, hydroxyl radical formation was greatly enhanced, up to eight-fold. We found that synthetically modulated derivatives of rutin exerted protective effects on $\mathrm{ONOO}^{-}$-mediated SERCA injury at low concentrations $(5-50 \mu \mathrm{mol} / \mathrm{l})$ and inhibitory at higher concentrations (100-250 $\mu \mathrm{mol} / \mathrm{l})$, (Viskupicova $e t$ al., 2009). Interestingly, the ability of rutin derivatives to inhibit SERCA activity in higher concentrations was not correlated with the prooxidant properties, probably due direct structural changes of SERCA by flavonoids.

Prooxidant or antioxidant properties of a particular flavonoid depend most expressively on its concentration. The furher could be associated with cell signaling by which flavonoids contribute to the co-ordination of cell functions.

\section{Health benefits of green tea to humans}

Tea is one of the most widely consumed beverages in the world. Polyphenolic compounds in green tea have recently received increased attention as preventive agents that may provide health benefits to humans. Green tea polyphenols include epigallocatechin-3-gallate (EGCG), epigallocatechin (EGC), epicatechin-3-gallate (ECG), and epicatechin (EC). The chemical structure of catechins is shown in Figure 3. The health benefits attributed to tea might result at least partly from the inhibitory effect of EGCG on $\mathrm{Na}^{+}, \mathrm{K}^{+}$-ATPase and $\mathrm{Ca}^{2+}$-ATPase activity (Ochiai et al., 2009).

EGCG is the most abundant of these catechins (Bettuzzi et al., 2006). It has been suggested to inhibit the activity of $\mathrm{Na}^{+}, \mathrm{K}^{+}$-ATPase in human erythrocyte ghosts (Rizvi \& Zaid, 2005), P-type ATPases (gastric $\mathrm{H}^{+}, \mathrm{K}^{+}$-ATPase and sarcoplasmic reticulum $\mathrm{Ca}^{2+}$-ATPase). Daily intake of EGCG by drinking green tea may provide physiological benefits to humans via inhibition of $\mathrm{Na}^{+}, \mathrm{K}^{+}-$ ATPase. EGCG and ECG exert their inhibitory effect on the membrane-bound $\mathrm{Na}^{+}, \mathrm{K}^{+}$-ATPase allosterically through interaction with plasma membrane phospholipid. The two galloyl-type catechins (EGCg and ECg) showed a stronger inhibitory effect on $\mathrm{Na}^{+}, \mathrm{K}^{+}$-ATPase than the other two catechins lacking galloyl groups (EGC and EC). EGCg and ECG have a greater affinity for membrane phospholipid than EGC and EC (Kamihara et al., 2008).

\section{Cancer chemoprotection by flavonoids}

Some flavonoids have been reported to reach levels of several micromolars in human blood plasma (Erlund $e t$ al., 2002). Flavonoids are believed to be cancer chemoprotective due to their ability to sensitize some cells into undergoing apoptosis, a process that has become 


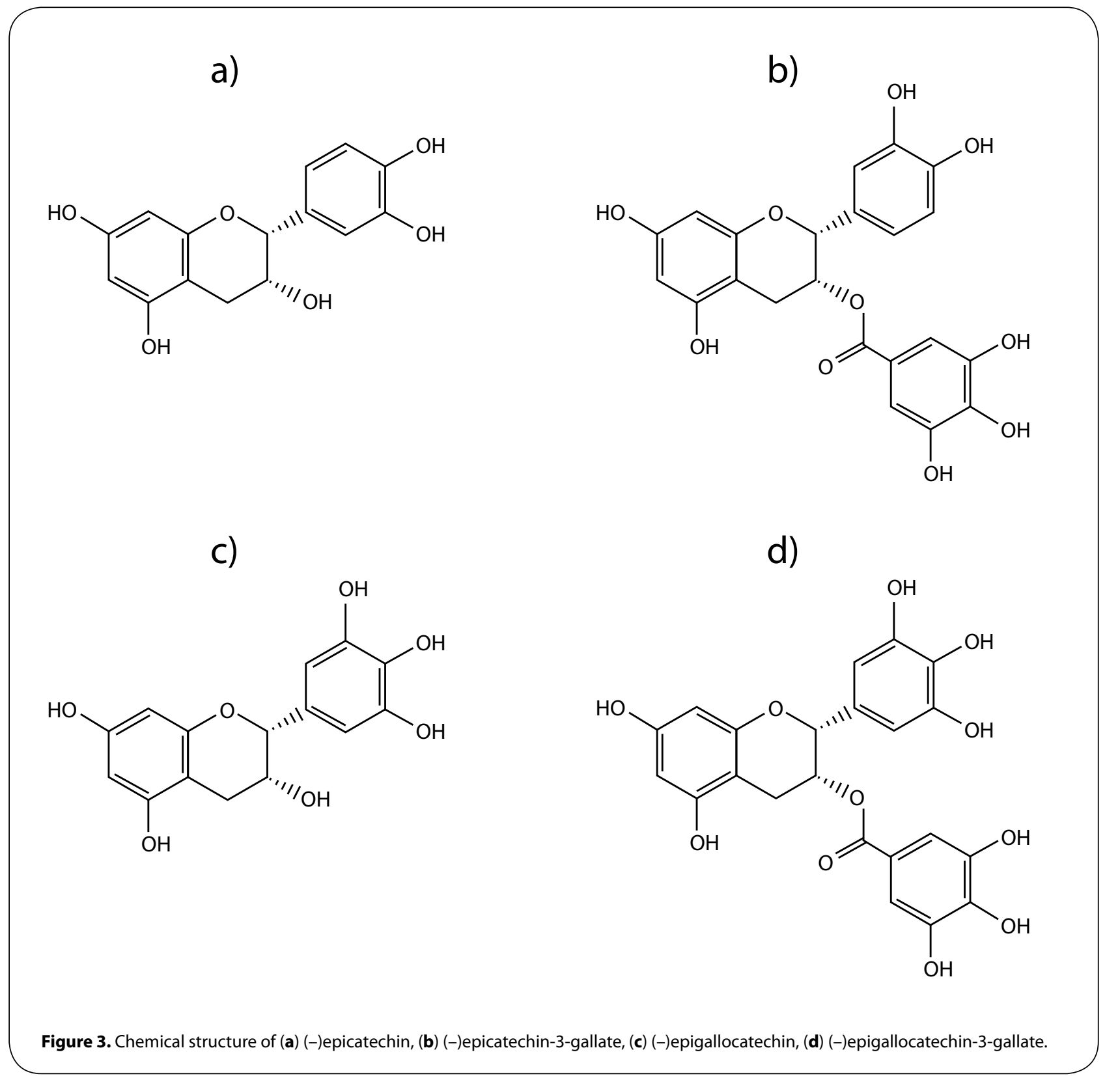

misregulated in many cancers. One hypothesis is that flavonoids are able to initiate apoptosis, especially in cancer cells, via a $\mathrm{Ca}^{2+}$-dependent mitochondrial pathway (Ogunbayo et al., 2008). This pathway can be activated through an exaggerated elevation of cytosolic $\left[\mathrm{Ca}^{2+}\right]$ and SERCA plays here an essential role by ameliorating such changes. Flavonoids (especially flavones) can inhibit the activity of $\mathrm{Ca}^{2+}$-ATPases isoforms SERCA1A and SERCA2B in the micromolar concentration range. Of the 25 flavonoids tested, 3,6-dihydroxyflavone (IC50, 4.6 $\mu \mathrm{M}$ ) and 3,30,40,5,7-pentahydroxyflavone (quercetin) (IC50, $8.9 \mu \mathrm{M})$ were the most potent inhibitors. The polyhydroxylation of flavones is important for inhibition, with hydroxylation at position 3 (for SERCA1A) and position 6 (for SERCA2B) being particularly relevant (Ogunbayo et al., 2008).
Natural flavonoid extract Pyc induced apoptosis in human mammary cancer cells, whereas normal mammary cells were not affected (Rodewald, 2002). Pyc is able to inhibit SERCA (Štrosová et al., 2006; Voss et al., 2008), which may be linked with its ability to induce apoptosis via an increase of tightly controlled cytosolic $\mathrm{Ca}^{2+}$. Similarly, other authors found an inhibiting effect of the anticancerogenic polyphenolic antioxidant curcumin against $\mathrm{Ca}^{2+}$-ATPase (Bilmen et al., 2001). Interestingly, the apoptosis of peripheral blood lymphocytes was on the contrary significantly reduced in patients with systemic lupus erythematosus treated with Pyc (Rodewald, 2002).

In recent years, research has shown that some flavonoids can trigger apoptosis through the modulation of a number of key cellular signaling pathways that can cause increased cellular levels of caspases and decreased 
levels of antiapoptotic factors such as Bcl-2-type proteins (Ramos, 2007; Selvendiran et al., 2006; Kuhar et al., 2006).

Green tea has been suggestive to be a rather effective cancer preventive beverage. Suganuma (2011) studied the effects of combining EGCG and anticancer drugs, focusing on inhibition of cell growth and induction of apoptosis. Synergistic enhancement of apoptosis, gene expression, and anticancer effects were found on using various combinations of EGCG and anticancer drugs, including the combination of (-)-epicatechin (EC) and curcumin (Suganuma et al., 2011).

Extracts of green tea and green tea polyphenols have exhibited inhibitory effects against the formation and development of tumors at different organ sites in animals (Yang \& Wang, 2010). These include animal models for skin, lung, oral cavity, esophagus, stomach, intestine, colon, liver, pancreas, bladder, mammary gland, and prostate cancers. In addition to suppressing cell proliferation, promoting apoptosis, and modulating signaling transduction, green tea polyphenols, especially EGCG, also inhibit cell invasion, angiogenesis, and metastasis (Yang \& Wang, 2010).

Flavonoids with inhibitory properties to $\mathrm{Ca}^{2+-}$-ATPase activity may represent agents with the ability to affect cellular processes known to be regulated by $\mathrm{Ca}^{2+}$, including apoptosis, decreased synthesis of ATP and free radical formation.

\section{Green tea flavonoids and brain dementia}

Green and black tea drinking (rich in a class of flavonoids named catechins) may help protect the aging brain and reduce the incidence of dementia like Alzheimer's disease (AD) and Parkinson's disease (PD). Neuroprotective/ neuroregenerative effects of green tea catechins were found to act not only as antioxidant metal chelators but also as modulators of intracellular neuronal signaling and metabolism, of cell survival/death genes, and mitochondrial function. Thus, these dietary compounds are receiving significant attention as therapeutic multifunctional cytoprotective agents that simultaneously manipulate various brain targets (Mandel et al., 2011).

\section{Cardioprotective effects of flavonoids}

Higher flavonoid intake from fruits and vegetables is associated with decreased risk for the development of cardiovascular disease. The mechanisms explaining this observation remain unclear, but current evidence suggests that flavonoids may exert their effects through affecting cardiovascular risk factors. Flavonoids improve endothelial function, inhibit low-density lipoprotein oxidation, decrease blood pressure and improve dyslipidemia in studies focused on cocoa, soy, and green and black tea. Some polyphenols in their purified form, including resveratrol, berberine and naringenin, exerted beneficial effects on dyslipidemia in humans and/or animals. In a mouse model of cardiovascular disease, naringenin treatment, through correction of dyslipidemia, hyperinsulinemia and obesity, attenuated atherosclerosis. Thus the beneficial effects of flavonoids on multiple risk factors may explain, in part, their observed beneficial effects on cardiovascular disease (Mulvihill \& Huff, 2010).

Several authors studied the effects of green and black tea or their individual components on the risk of cardiovascular diseases. Regular consumption of black and green tea may reduce the risk of cardiovascular disease. The cardiovascular health benefits of drinking tea are thought to be largely due to flavonoids. Isolated flavonoids found in tea have also been consistently shown to inhibit the development of atherosclerosis in animal models (Hodgson \& Croft, 2010).

Molecular mechanisms of cardiovascular protection of green tea polyphenols, particularly EGCG, focusing on anti-oxidative and anti-inflammatory effects, were studied by Tipoe (2007). The most active component in green tea was found to be EGCG. Studies have shown that EGCG protects cellular damage by inhibiting DNA damage and oxidation of low density lipoproteins (LDL). One of the protective properties of EGCG is its ability to scavenge free radicals. EGCG was also able to reduce the inflammatory response associated with local tissue injuries, such as hepatocellular necrosis in acute liver injury induced by carbon tetrachloride. The protective effect of EGCG is due to its ability to decrease lipid peroxidation, oxidative stress and the production of nitric oxide (NO) radicals by inhibiting the expression of inducible nitric oxide synthase (iNOS). EGCG also ameliorates the overproduction of pro-inflammatory cytokines and mediators, reduces the activity of nuclear factor kappalight-chain-enhancer of activated B cells (NF-kappa $\mathrm{B}$ ) and transcription factor AP-1 and the subsequent formation of peroxynitrite with $\mathrm{NO}$ and reactive oxygen species. Thus EGCG effectively mitigated cellular damage by lowering the inflammatory reaction and reducing lipid peroxidation and NO-generated radicals leading to oxidative stress. Green tea is proposed to be a dietary supplement in the prevention of cardiovascular diseases, in which oxidative stress and proinflammation are the principal causes.

Other studies concerning flavonoid benefits were focused on cardiac hypertrophy. Cardiac hypertrophy is a pathological response of the heart to chronic pressure or volume overload. Cardiac hypertrophy is a risk factor for ischemic heart disease, arrhythmia, and sudden death. It is therefore important to determine the mechanism of the development of cardiac hypertrophy and prevent or treat it. Chronic low level of cardiac myocyte apoptosis was proposed to be a causal component in the pathogenesis of heart failure (Sheng et al., 2007).

Many antioxidants have shown inhibitory effects on cardiac hypertrophy in vitro and in vivo induced by ROS (Sheng et al., 2007; Tsujimoto, 2005). EGCG and green tea were found to exert protective effects against cardiovascular diseases (Chyu et al., 2004; Townsend et al., 2004). Green tea extracts and EGCG protected cardiomyocytes against ischemia/reperfusion-induced apoptotic cell death both in vivo and in vitro and effectively inhibited 
cardiac hypertrophy in mice and rats (Sheng et al., 2007; Li et al., 2006).

The inhibitory effects of EGCG on cardiac hypertrophy was tested by Sheng et al. (2007), and it was found that EGCG inhibited cardiac myocyte apopotosis and oxidative stress in pressure overload induced cardiac hypertrophy. EGCG also prevented cardiomyocyte apoptosis from oxidative stress in vitro.

Summarizing the above mentioned facts, we conclude that mechanisms of beneficial effects of flavonoids in cardiovascular diseases are based mainly on their antioxidant and antiinflammatory properties. On the other hand, the mechanism of cardiac hypethrophy prevention by flavonoids is suggested to operate via protection against apoptotic cell death of cardiomyocytes.

\section{Doxorubicin-induced cardiomyocyte injury}

The effectiveness of the anticancer drug doxorubicin is severely limited by its cardiac side-effects related to cardiomyopathy and heart failure (Clement, 2009). The putative mechanism of doxorubicin-induced cardiotoxicity is the generation of reactive oxygen species during its intracellular metabolism (Choi et al., 2007). An increasing number of studies suggest that disruption of calcium $\left(\mathrm{Ca}^{2+}\right)$ homeostasis is another important cause for doxorubicin-mediated cardiotoxicity. Precise $\mathrm{Ca}^{2+}$ handling in cardiomyocytes is crucial for excitation-contraction coupling, the process that enables the heart to function effectively as a pump. Doxorubicin was reported to regulate the functions of L-type $\mathrm{Ca}^{2+}$ channels, $\mathrm{Na}^{+} / \mathrm{Ca}^{2+}$ exchanger, SERCA2 gene transcription and ryanodine receptors, thus modulating heart contractile function. (Zheng et al., 2011)

Doxorubicin-induced cardiac myopathy was found to be reduced by antioxidant properties of EGCG. (Bast et al., 2007). In addition, EGCG protection has also other aspects. One such aspect is its protein-binding ability which can help in the protection of cellular proteins against oxidative damage by reactive oxygen species (Hagerman et al., 2003). As proteins are targets of oxidative damage, the interference of oxidative damage by EGCG may have a potential role in attenuating the development of cardiomyopathy.

Oxidative stress may also influence $\mathrm{Ca}^{2+}$ homeostasis by directly inducing mitochondrial permeability transition with modifications in mitochondrial calcium transport. The changes in calcium transport can further lead to tissue injury, cell killing and impaired cardiac contraction (Schimmel et al., 2004). EGCG significantly increased cell viability and protected against apoptosis in doxorubicin-treated cells.

The effect of EGCG on $\mathrm{Ca}^{2+}$ handling was studied in cardiac myocytes and it was found to protect myocytes against doxorubicin-induced intracellular calcium depletion (Zheng et al., 2011).

In conclusion, EGCG-reduced cardiomyopathy after doxorubicin administration may be caused by several mechanisms: by antioxidant propeties of EGCG, its protein binding ability, protection against apoptosis of cardiac myocytes and protection against intracellular calcium depletion. In this respect, SERCA may be involved in the above mentioned protection, as flavonoids are able to bind to SERCA, change its activity and thus also the intracellular calcium level.

\section{SERCA dysfunction in diseases, posttranlational and conformational alterations}

Conformational alterations of $\mathrm{Ca}^{2+}$-ATPases were studied in several diseases. Studies of ischemia/reperfusion in cardiac muscle and more recently in skeletal muscle showed that impaired sarcoplasmic reticulum (SR) function and $\mathrm{Ca}^{2+}$ homeostasis may be involved in the etiology of ischemia/reperfusion injury. Ischemia-induced structural changes in SR $\mathrm{Ca}^{2+}$-ATPase are associated with reduced enzyme activity in rat muscle. Experiments with prolonged ischemia in rat skeletal muscle revealed that structural modifications to SR $\mathrm{Ca}^{2+}$-ATPase can explain the alterations in $\mathrm{Ca}^{2+}$-ATPase activity that occur with ischemia (Tuplin et al., 2001). They found that reduction in maximal SR $\mathrm{Ca}^{2+}$-ATPase activity in SR vesicles with ischemia was related to structural modification in the region of the nucleotide binding domain.

SERCA plays a key role in the relaxation of smooth, cardiac and skeletal muscle through the transport of cytosolic $\mathrm{Ca}^{2+}$ into SR or ER (MacLennan et al., 1997; East, 2000; Sharov et al., 2006). However also other physiological and pathological processes are associated with an abnormal activity and expression of SERCA, such as cell proliferation, apoptosis, Brody and Darier disease and cancer (Sharov et al., 2006).

By reacting with superoxide anion, the less reactive NO forms the more thiol reactive oxidant peroxynitrite, which in turn can adduct glutathione (GSH) to SERCA cysteine thiols. The reaction of NO with superoxide anion is required, as indicated by the fact that mouse arteries in which superoxide dismutase is overexpressed relax less to NO (Adachi et al., 2004). The majority of GSH on SERCA is bound to the most reactive thiol on cysteine-674, and mutation of this single cysteine prevents not only formation of most GSH adducts but also stimulation of $\mathrm{Ca}^{2+}$ uptake by NO. Tong et al. (2010) reported prevention of such stimulation of $\mathrm{Ca}^{2+}$ by Cys674 mutation associated with decrease of cell migration. NO function is impaired in a variety of cardiovascular diseases, including diabetic vascular disease and atherosclerosis, which are associated with irreversible oxidation of SERCA cysteine-674 (Tong et al., 2010).

In atherosclerotic arteries, NO can not stimulate SERCA activity because of the irreversible oxidation of the cysteine-674 thiol caused by the high levels of oxidants accompanying the disease (Adachi et al., 2004).

Viner et al. (1999a) reported inactivation of SERCA by higher, pathological levels of NO or peroxynitrite. Such inactivation can be physiologically detrimental as a reduced function of SERCA has been associated with changed cellular response to apoptotic stimuli and also 
with an increased risk for cancer (Vanoverberghe et al., 2004).

The increase of RNS in vascular diseases (such as atherosclerosis or diabetes) irreversibly oxidizes Cys674 or nitrate tyrosine residues at Tyr 296-Tyr297, which is associated with loss of SERCA function (Adachi et al., 2010). Oxidative inactivation of target proteins for $\mathrm{NO}$ can be associated with the pathogenesis of cardiovascular diseases. Oxidative inactivation of SERCA is also implicated in dysregulation of smooth muscle migration, promotion of platelet aggregation and impairment of cardiac function, which can be associated with restenosis, pathological angiogenesis, thrombosis, as well as heart failure. Specific irreversible oxidative modifications consisting of sulfonylation at cysteine 674 and nitration at tyrosines 294/295 were found also in myocyte contractile dysfunction in the mouse heart mediated by reactive oxygen species (Lancel et al., 2010).

NO-induced vasodilatation is impaired in patients with a variety of cardiovascular diseases, including diabetes, hypercholesterolemia ( $\mathrm{HC}$ ) and atherosclerosis (Adachi et al., 2002, 2004; Williams et al., 1996). SERCA 1 activity was markedly decreased in HC, although SERCA 2 protein expression did not change (Adachi et al., 2001). The abnormal SERCA function in the HC rabbit aorta can be at tributed to extensive oxidant-induced tyrosine nitration and thiol oxidation of SERCA (Adachi et al., 2002; Ying et al., 2008). Both tyrosine nitration and thiol oxidation may be attributed to excess production of peroxynitrite, the chemical product of $\mathrm{NO}$ and superoxide (Adachi et al., 2002; Ying et al., 2008).

In diabetic and insulin-treated diabetic Wistar rats, correlation was found between increase in tyrosine nitration of aortic SERCA2b and impaired aortic relaxation to acetylcholine, characteristic for the diabetic state (Adachi et al., 2001). Prolonged treatment of the diabetic rat with insulin also impaired aortic relaxations that were attributed to dysfunction of the smooth muscle and were caused, at least in part, by SERCA dysfunction (Adachi et al., 2001). In the streptozotocin-induced diabetic rat model, increased plasma angiotensin II and peroxynitrite levels impaired cyclic GMP-independent aortic relaxation, apparently by inhibiting SERCA (Taguchi et al., 2007).

Additionally, the diabetic $\mathrm{HC}$ pig aorta contained SERCA2b in which the cysteine-674 thiol was oxidized, and this was prevented in pigs treated with insulin. Interestingly, the irreversible oxidation of SERCA in the diabetic pig aorta corresponded to a less intact $110 \mathrm{kDa}$ SERCA protein and a lower molecular mass SERCA protein of approximately $70 \mathrm{kDa}$. These studies suggest that SERCA degradation is associated with its oxidation and may play a role in progression of diabetic vascular disease (Ying et al., 2008).

SERCA2 in platelets from patients with type 2 diabetes mellitus showed increased tyrosine nitration accompanied by inactivation of SERCA, elevated platelet free $\mathrm{Ca}^{2+}$ levels, and activated $\mu$-calpain. The tyrosine nitration of SERCA2 and the activation of $\mu$-calpain in platelets from healthy volunteers could be evoked by peroxynitrite in vitro, implicating the importance of oxidation of SERCA in diabetic patients (Randriamboavonjy et al., 2008).

According to our recent results, development of adjuvant arthritis in an animal model was related to modulation of SERCA activity and correlated with oxidation of cysteine and nitration of tyrosine. We concluded that nitric oxide may regulate cytoplasmic $\mathrm{Ca}^{2+}$ level through conformational alterations in the transmembrane $\mathrm{Ca}^{2+}$ binding domain of SERCA (Strosova et al., 2011).

Biological aging leads to oxidation and nitration of SERCA at Cys and Tyr residues (Restall et al., 1986), accompanied by partial inactivation of SERCA activity and to a conformationally altered nucleotide binding site (Chen et al, 1999). Sharov et al. (2006) found that age-dependent loss of Cys residues may be partially responsible for the age-dependent decrease of the specific Ca-ATPase activity. Biological aging resulted in partial modification of Cys residues 377, 498, 525, 561, 614, 636, $674,675,774,938$. In the SERCA2a from aged slow-twich skeletal muscle (Viner et al., 1999b) and heart (Knyushko et al., 2005) accumulation of 3-nitrotyrosine (3-NT) at positions 294, 295, located at the lumen-membrane interface of the transmembrane helix M4, and at position 753 was found.

Summarizing SERCA dysfunction in the above mentionad diseases, it can be concluded that cysteine sulfhydryls, mainly in Cys 674 and tyrosine nitration usually at positions 294,295 , are the main posttranslational alterations of SERCA responsible for modulation of its activity, correlating with pathological disorders. Conformational alterations of SERCA in cytosolic nucleotide (ATP-binding) sites observed both in aging and in ischemia/reperfusion were found in cardiac and skeletal muscles, while conformational changes in the transmembrane domain of SERCA were associated with adjuvant arthritis. Several authors concluded that SERCA oxidative post translational modifications contributed to the pathophysiology of individual diseases and that preservation of SERCA function and/or increasing expression of SERCA may be a novel strategy against diseases associated with oxidative stress (Adachi et al., 2010, Lancet et al., 2010, Tong et al., 2010, Inesi et al., 2005).

\section{SERCA as a therapeutic target to prevent vascular disease development}

Preventing SERCA oxidation and/or providing new SERCA protein may improve vascular disease development as indicated by several examples. In a study of impaired endothelium-dependent relaxations in the $\mathrm{HC}$ rabbit aorta, the antioxidant t-butylhydroxytoluene (BHT) dramatically improved the smooth muscle response to NO. The beneficial effects of the antioxidant depended on improvement of the response to NO in the smooth muscle cells (SMC) (Adachi et al., 2002). BHT also restored the decreased aortic SERCA activity in HC and decreased tyrosine-nitrated SERCA without changing SERCA protein expression. These beneficial effects may depend on 
decreasing the direct effects of ROS/RNS on SERCA that are augmented in HC (Adachi et al., 2002).

Another example is improvement of endotheliumdependent relaxation which precedes the regression of atherosclerosis caused by cholesterol lowering or antioxidant treatments. This suggests that improvement of the response to NO can limit the progression of atherosclerosis. The improvement in SMC function is associated with decreased oxidative modification of SERCA protein and improved $\mathrm{Ca}^{2+}$ uptake activity. This suggests that preservation of SERCA function may be regarded as a new target for the treatment of impaired vascular function.

In the streptozotocin-induced diabetic rat model, elevated plasma angiotensin II levels were improved by treatment with the angiotensin-converting enzyme inhibitor enalapril. As angiotensin II is a powerful inducer of NADPH oxidase, it is likely that enalapril helps prevent the diabetes-related impairment of SERCA function in the aorta by suppressing ROS formation (Taguchi et al. 2007).

The enhanced tyrosine nitration and inactivation of SERCA2 in platelets from type 2 diabetic patients were restored by treating the patients with rosiglitazone. This was accompanied by improvements in platelet $\left[\mathrm{Ca}^{2+}\right] \mathrm{i}$ and aggregation, indicating the importance of SERCA oxidation in platelet function in diabetic patients. Rosiglitazone also partially restored inhibition of platelet aggregation by NO, consistent with improvements in SERCA redox function (Randriamboavonjy et al., 2008).

Nitrotyrosine, an indirect marker of peroxinitrite, was markedly increased in aortic smooth muscle from diabetic rats, while chronic enalapril administration reduced this increase. In streptozotocin-induced diabetic rats, angiotensin II production may lead to the generation of peroxynitrite and this may in turn trigger dysfunction of vascular smooth muscle SERCA (Taguchi et al., 2007).

$\mathrm{NO}$ relaxes arteries, in part by stimulating $\mathrm{Ca}^{2+}$ uptake via SERCA in aortic smooth muscle, while HC impairs SERCA function and the response to NO. HC induces oxidative stress, which can impair SERCA function. Antioxidant BHT reversed impaired smooth muscle SERCA function in $\mathrm{HC}$, which correlated with improved relaxation to NO (Adachi et al., 2002).

\section{Conclusions}

Both physiological and pathophysiological levels of ROS/ RNS regulate cell functions by direct modifications of SERCA. This may contribute to the understanding of the way in which ROS/RNS regulate cell function and may also suggest new strategies to prevent vascular diseases and development of cancer.

Flavonoids are able to initiate apoptosis, especially in cancer cells, via a $\mathrm{Ca}^{2+}$-dependent mitochondrial pathway, which can be activated through an exaggerated elevation of cytosolic $\left[\mathrm{Ca}^{2+}\right]_{\mathrm{i}}$ and at least partially via sarcoplasmic/endoplasmic reticulum $\mathrm{Ca}^{2+}$-ATPases. Thus flavonoids may be a useful tool for preventing cancer development.
On the other hand, decrease of apoptosis is required in some cases. In cardiac hypertrophy in vitro and in vivo induced by ROS/RNS, the changes in calcium transport can further lead to tissue injury, cell killing and impaired cardiac contraction. Some flavonoids significantly increased cell viability and protected against cardiomyocyte apoptosis, via preservation of SERCA function, which can be a novel strategy against cardiovascular diseases associated with oxidative stress.

\section{Acknowledgement}

This work was supported by The Agency of the Ministry of Education, Science, Research and Sport of the Slovak Republic for the Structural Funds of EU, OP R\&D of ERDF in the frame of the Project „Evaluation of natural substances and their selection for prevention and treatment of lifestyle diseases (ITMS 26240220040).

\section{REFERENCES}

Adachi T, Matsui R, Weisbrod RM, Najibi S, Cohen RA. (2001). Reduced sarco/ endoplasmic reticulum $\mathrm{Ca}(2+)$ uptake activity can account for the reduced response to NO, but not sodium nitroprusside, in hypercholesterolemic rabbit aorta. Circulation 104: 1040-5.

Adachi T, Matsui R, Xu S, Kirber M, Lazar HL, Sharov VS, Schöneich C, Cohen RA. (2002). Antioxidant improves smooth muscle sarco/endoplasmic reticulum $\mathrm{Ca}\left({ }^{2+}\right)$-ATPase function and lowers tyrosine nitration in hypercholesterolemia and improves nitric oxide-induced relaxation. Circ Res 90: 1114-1121.

Adachi T, Weisbrod RM, Pimentel DR, Ying J, Sharov VS, Schöneich C, Cohen RA. (2004). S-Glutathiolation by peroxynitrite activates SERCA during arterial relaxation by nitric oxide. Nat Med 10: 1200-1207.

Adachi T. (2010). Modulation of vascular sarco/endoplasmic reticulum calcium ATPase in cardiovascular pathophysiology. Adv Pharmacol 59: 16595. Review.

Amić D, Davidović-Amić D, Beslo D, Rastija V, Lucić B, Trinajstić N. (2007). SAR and QSAR of the antioxidant activity of flavonoids. Curr Med Chem 14: $827-$ 45. Review.

Bast A, Haenen GR, Bruynzeel AM, Van der Vijgh WJ. (2007). Protection by flavonoids against anthracycline cardiotoxicity: from chemistry to clinical trials. Cardiovasc Toxicol 7: 154-159. Review.

Bettuzzi S, Brausi M, Rizzi F, Castagnetti G, Peracchia G, Corti A. (2006). Chemoprevention of human prostate cancer by oral administration of green tea catechins in volunteers with high-grade prostate intraepithelial neoplasia: a preliminary report from a one-year proof-of-principle study. Cancer Res 66: 1234-40.

Bigelow DJ. (2009). Nitrotyrosine-modified SERCA2: a cellular sensor of reactive nitrogen species. Pflugers Arch 457: 701-710.

Bilmen JG, Khan SZ, Javed MH, Michelangeli F. (2001). Inhibition of the SERCA $\mathrm{Ca}^{2+}$ pumps by curcumin. Curcumin putatively stabilizes the interaction between the nucleotide-binding and phosphorylation domains in the absence of ATP. Eur J Biochem 268: 6318-6327.

Burda S, Oleszek W. (2001). Antioxidant and antiradical activities of flavonoids. J Agric Food Chem 49: 2774-2779.

Cao G, Sofic E, Prior RL. (1997). Antioxidant and prooxidant behavior of flavonoids: structure-activity relationships. Free Radic Biol Med 22: 749-60.

Clement Y. (2009). Can green tea do that? A literature review of the clinical evidence. Prev Med 49: 83-87. Review.

Chen B, Jones TE, Bigelow DJ. (1999). The nucleotide-binding site of the sarcoplasmic reticulum Ca-ATPase is conformationally altered in aged skeletal muscle. Biochemistry 38:14887-14896.

Choi EH, Chang HJ, Cho JY, Chun HS. (2007). Cytoprotective effect of anthocyanins against doxorubicin-induced toxicity in $\mathrm{H} 9 \mathrm{c} 2$ cardiomyocytes in relation to their antioxidant activities. Food Chem Toxicol 45: 1873-1881. 
Chyu KY, Babbidge SM, Zhao X, Dandillaya R, Rietveld AG, Yano J, Dimayuga P, Cercek B, Shah PK. (2004). Differential effects of green tea-derived catechin on developing versus established atherosclerosis in apolipoprotein E-null mice. Circulation 109: 2448-2453.

Cohen RA and Adachi T. (2006). Nitric-oxide-induced vasodilatation: Regulation by Physiologic S-glutathiolation and pathologic oxidation of the sarcoplasmic endoplasmic reticulum calcium ATPase. TCM 16: 109-114.

Croft KD. (2006). The chemistry and biological effects of flavonoids and phenolic acids. Ann NY Acad Sci 854: 435-442.

Dremina ES, Sharov VS, Davies MJ, Schöneich C. (2007). Oxidation and inactivation of SERCA by selective reaction of cysteine residues with amino acid peroxides. Chem Res Toxicol 20: 1462-1469.

Drieu K. (1986). Preparation and definition of the extract of Ginkgo biloba. Presse Médicale 15: 1455-1457.

Dyer JL, Khan SZ, Bilmen JG, Hawtin SR, Wheatley M, Javed MU, Michelangeli F. (2002). Curcumin: a new cell-permeant inhibitor of the inositol 1,4,5-trisphosphate receptor. Cell Calcium 31: 45-52.

East JM. (2000). Sarco(endo)plasmic reticulum calcium pumps: recent advances in our understanding of structure/function and biology. Mol Membr Biol 17: 189-200.

Erlund I, Silaste ML, Alfthan G, Rantala M, Kesäniemi YA, Aro A. (2002). Plasma concentrations of the flavonoids hesperetin, naringenin and quercetin in human subjects following their habitual diets, and diets high or low in fruit and vegetables. Eur J Clin Nutr 56: 891-898.

Hagerman AE, Dean RT, Davies MJ. (2003). Radical chemistry of epigallocatechin gallate and its relevance to protein damage. Arch Biochem Biophys 14: 115-120.

Hanasaki Y, Ogawa S, Fukui S. (1994). The correlation between active oxygens scavenging and antioxidative effects of flavonoids. Free Radic Biol Med 16: 845-50.

Heim KE, Tagliaferro AR, Bobilya DJ. (2002). Flavonoid antioxidants: chemistry, metabolism and structure-activity relationships. J Nutr Biochem 13: 572-584.

Hodgson JM, Croft KD. (2010). Tea flavonoids and cardiovascular health. Mol Aspects Med 31: 495-502. Review.

Inesi G, Hua S, Xu C, Ma H, Seth M, Prasad AM, Sumbilla C. (2005). Studies of Ca2+ ATPase (SERCA) inhibition. J Bioenerg Biomembr 37: 365-8. Review.

Ishige K, Schubert D, Sagara Y. (2001). Flavonoids protect neuronal cells from oxidative stress by three distinct mechanisms. Free Radical Biology \& Medicine 30: 433-446.

Kamihira M, Nakazawa H, Kira A, Mizutani Y, Nakamura M, Nakayama T. (2008). Interaction of tea catechins with lipid bilayers investigated by a quartz-crystal microbalance analysis. Biosci Biotechnol Biochem 72: 13721375.

Knyushko TV, Sharov VS, Williams TD, Schöneich C. and Bigelow DJ. (2005). 3-Nitrotyrosine-modification of SERCA2a in the aging heart: a distinct signature of cellular redox environment. Biochemistry 44: 13071-13081.

Kuhar M, Sen S, Singh N. (2006). Role of mitochondria in quercetin-enhanced chemotherapeutic response in human non-small cell lung carcinoma H-520 cells. Anticancer Res 26: 1297-1303.

Lancel S, Qin F, Lennon SL, Zhang J, Tong X, Mazzini MJ, Kang YJ, Siwik DA, Cohen RA, Colucci WS. (2010). Oxidative posttranslational modifications mediate decreased SERCA activity and myocyte dysfunction in Galphaqoverexpressing mice. Circ Res 107: 228-232.

Leung HW, Kuo CL, Yang WH, Lin $\mathrm{CH}$, Lee HZ. (2006). Antioxidant enzymes activity involvement in luteolin-induced human lung squamous carcinoma CH27 cell apoptosis. Eur J Pharmacol 534: 12-18.

MacLennan DH, Rice W J. and Green NM. (1997). The mechanism of Ca2+ transport by sarco(endo)plasmic reticulum Ca2+-ATPases. J Biol Chem 272: 28815-28828.

Mandel SA, Amit T, Weinreb O, Youdim MB. (2011). Understanding the broadspectrum neuroprotective action profile of green tea polyphenols in aging and neurodegenerative diseases. J Alzheimers Dis 25(2): 187-208.

Martín MA, Serrano AB, Ramos S, Pulido MI, Bravo L, Goya L. (2010). Cocoa flavonoids up-regulate antioxidant enzyme activity via the ERK1/2 pathway to protect against oxidative stress-induced apoptosis in HepG2 cells. J Nutr Biochem 21: 196-205.

Mulvihill EE, Huff MW. (2010). Antiatherogenic properties of flavonoids: implications for cardiovascular health. Can J Cardio/ 26(Suppl A): 17A-21A. Review.
Nagata H, Takekoshi S, Takagi T, Honma T, Watanabe K. (1999). Antioxidative action of flavonoids, quercetin and catechin, mediated by the activation of glutathione peroxidase. Tokai J Exp Clin Med 24: 1-11.

Ochiai H, Takeda K, Soeda S, Tahara Y, Takenaka H, Abe K, Hayashi Y, Noguchi S, Inoue M, Schwarz S, Schwarz W, Kawamura M. (2009). Epigallocatechin3-gallate is an inhibitor of $\mathrm{Na}+\mathrm{K}(+)$-ATPase by favoring the E1 conformation. Biochem Pharmacol 78: 1069-74.

Ogunbayo OA, Harris RM, Waring RH, Kirk CJ, Michelangeli F. (2008). Inhibition of the sarcoplasmic/endoplasmic reticulum Ca2+-ATPase by flavonoids: a quantitative structure-activity relationship study. IUBMB Life 60: 853-858.

Pacher P, Beckman JS, Liaudet L. (2007). Nitric oxide and peroxynitrite in health and disease. Physiol Rev 87: 315-424. Review.

Packer L, Rimbach G, Virgili F. (1999). Antioxidant activity and biologic properties of a procyanidin-rich extract from pine (Pinus maritima) bark, pycnogenol. Free Radical Biology \& Medicine 27: 704-724.

Procházková D, Boušová I, Wilhelmová N. (2011). Antioxidant and prooxidant properties of flavonoids. Fitoterapia 82: 513-523.

Ramos S. (2007). Effects of dietary flavonoids on apoptotic pathways related to cancer chemoprevention. J Nutr Biochem 18: 427-442.

Randriamboavonjy V, Pistrosch F, Bölck B, Schwinger RH, Dixit M, Badenhoop K, Cohen RA, Busse R, Fleming I. (2008). Platelet sarcoplasmic endoplasmic reticulum $\mathrm{Ca}^{2+}$-ATPase and mu-calpain activity are altered in type 2 diabetes mellitus and restored by rosiglitazone. Circulation 117: 52-60.

Restall CJ, Coke M, Phillips E, Chapman D. (1986). Derivative spectroscopy of tryptophan fluorescence used to study conformational transitions in the $\left(\mathrm{Ca}^{2+} \mathrm{Mg}^{2+}\right)$-adenosine triphosphatase of sarcoplasmic reticulum. Biochim Biophys Acta 874: 305-311.

Rice-Evans CA, Miller NJ, Paganga G. (1996). Structure-antioxidant activity relationships of flavonoids and phenolic acids. Free Radic Biol Med 20:933-956.

Rizvi SI, Zaid MA. (2005). Impairment of sodium pump and Na/H exchanger in erythrocytes from non-insulin dependent diabetes mellitus patients: effect of tea catechins. Clin Chim Acta 354: 59-67.

Rohdewald P. (2002). A review of the French maritime pine bark extract (Pycnogenol), a herbal medication with a diverse clinical pharmacology. Review. International Journal of Clinical Pharmacology and Therapeutics 40: 158-168.

Sastre J, Millan A, De La Asuncion JG, Pla JG, Pallardo FV, O' Connor E, Martin JA, Droy-Lefaix MT, Viña J. (1998). A Ginkgo biloba extract (EGb 761) prevents mitochondrial aging by protecting against oxidative stress. Free Radical Biology \& Medicine 24: 298-304.

Schroeter H, Williams RJ, Matin R, Iversen L, Rice-Evans CA. (2000). Phenolic antioxidants attenuate neuronal cell death following uptake of oxidized low-density lipoprotein. Free Radical Biology \& Medicine 29: 1222-1233.

Schimmel KJ, Richel DJ, van den Brink RB, Guchelaar HJ. (2004). Cardiotoxicity of cytotoxic drugs. Cancer Treat Rev 30: 181-191. Review.

Selvendiran K, Koga H, Ueno T, Yoshida T, Maeyama M, Torimura T, Yano H, Kojiro M, Sata M. (2006). Luteolin promotes degradation in signal transducer and activator of transcription 3 in human hepatoma cells: an implication for the antitumor potential of flavonoids. Cancer Res 66: 4826-4834.

Sharov VS, Dremina ES, Galeva NA, Williams TD, Schöneich C. (2006). Quantitative mapping of oxidation-sensitive cysteine residues in SERCA in vivo and in vitro by HPLC-electrospray-tandem MS: selective protein oxidation during biological aging. Biochem J 394: 605-615.

Sheng R, Gu ZL, Xie ML, Zhou WX, Guo CY. (2007). EGCG inhibits cardiomyocyte apoptosis in pressure overload-induced cardiac hypertrophy and protects cardiomyocytes from oxidative stress in rats. Acta Pharmacol Sin 28: 191-201.

Shoshan V, MacLennan DH. (1981). Quercetin interaction with the $\left(\mathrm{Ca}^{2++}\right.$ $\left.\mathrm{Mg}^{2+}\right)$-ATPase of sarcoplasmic reticulum. J Biol Chem 256: 887-892.

Štrosová M, Karlovská J, Balgavý P, Horáková L. (2006). Effect of some antioxidants on sarcoplasmic reticulum $\mathrm{Ca}^{2+}$-ATPase activity from rabbit skeletal muscle. Neuroendocrinology Letters 27: 164-167.

Štrosová MK, Karlovská J, Žižková P, Kwolek-Mirek M, Poništ S, Spickett CM, Horáková '́. (2011). Modulation of sarcoplasmic/endoplasmic reticulum $\mathrm{Ca}(2+)$-ATPase activity and oxidative modification during the development of adjuvant arthritis. Arch Biochem Biophys 511: 40-47.

Štrosová M, Škuciová M., Horáková L. (2005). Oxidative damage to $\mathrm{Ca}^{2+}$ ATPase sarcoplasmic reticulum by $\mathrm{HOCl}$ and $\mathrm{HNE}$ and protective effect of some antioxidants. Biofactors 23: 111-116. 
Suganuma M, Saha A, Fujiki H. (2011). New cancer treatment strategy using combination of green tea catechins and anticancer drugs. Cancer Sci 102: 317-323. Review.

Sun C, Fu J, Chen J, Jiang L, Pan Y. (2010). On-line HPLC method for screening of antioxidants against superoxide anion radical from complex mixtures. $J$ Sep Sci 33: 1018-23.

Taguchi K, Kobayashi T, Hayashi Y, Matsumoto T, Kamata K. (2007). Enalapril improves impairment of SERCA-derived relaxation and enhancement of tyrosine nitration in diabetic rat aorta. Eur J Pharmacol 556: 121-128.

Taubert D, Breitenbach T, Lazar A, Censarek P, Harlfinger S, Berkels R, Klaus W, Roesen R. (2003). Reaction rate constants of superoxide scavenging by plant antioxidants. Free Radic Biol Med 35: 1599-1607.

Tipoe GL, Leung TM, Hung MW, Fung ML. (2007). Green tea polyphenols as an antioxidant and anti-inflammatory agent for cardiovascular protection. Cardiovasc Hematol Disord Drug Targets 7: 135-144. Review.

Tong X, Evangelista A, Cohen RA. (2010). Targeting the redox regulation of SERCA in vascular physiology and disease. Curr Opin Pharmacol 10: 133-138.

Tsujimoto I, Hikoso S, Yamaguchi O, Kashiwase K, Nakai A, Takeda T, Watanabe T, Taniike M, Matsumura Y, Nishida K, Hori M, Kogo M, Otsu K. (2005). The antioxidant edaravone attenuates pressure overload-induced left ventricular hypertrophy. Hypertension 45: 921-926.

Townsend PA, Scarabelli TM, Pasini E, Gitti G, Menegazzi M, Suzuki H, Knight RA, Latchman DS, Stephanou A. (2004). Epigallocatechin-3-gallate inhibits STAT-1 activation and protects cardiac myocytes from ischemia/reperfusion-induced apoptosis. FASEB J 18:1621-1623.

Tupling R, Green H, Senisterra G, Lepock J, McKee N. (2001). Ischemia-induced structural change in SR Ca2+-ATPase is associated with reduced enzyme activity in rat muscle. Am J Physiol Regul Integr Comp Physiol 281: R1681-1688.

Vanoverberghe $K$, Vanden Abeele F, Mariot P, Lepage G, Roudbaraki M, Bonnal JL, Mauroy B, Shuba Y, Skryma R. and Prevarskaya N. (2004). Ca ${ }^{2+}$ homeostasis and apoptotic resistance of neuroendocrine-differentiated prostate cancer cells. Cell Death Differ 11: 321-330.
Viner, R. I., Ferrington, D. A., Williams, T. D., Bigelow, D. J. and Schöneich, C (1999a) Protein modification during biological aging: selective tyrosine nitration of the SERCA2a isoform of the sarcoplasmic reticulum $\mathrm{Ca}^{2+}{ }_{-}$ATPase in skeletal muscle. Biochem J 340: 657-669.

Viner RI, Williams TD. and Schöneich C (1999b) Peroxynitrite modification of protein thiols: oxidation, nitrosilation, and S-glutathiolation of functionally important cysteine residue(s) in the sarcoplasmic reticulum Ca-ATPase. Biochemistry 38: 12408-12415.

Viskupičová J, Štrosová M, Šturdík E, Horáková L. (2009). Modulating effect of flavonoids and their derivatives on sarcoplasmic reticulum $\mathrm{Ca}^{2+}$-ATPase oxidized by hypochloric acid and peroxinitrite. Neuroendocrinology Letters 30: 148-151.

Voss P, Engels M, Štrosova M, Grune T, Horakova L'. (2008). Protective effect of antioxidants against sarcoplasmic reticulum (SR) oxidation by Fenton reaction, however without prevention of Ca-pump activity. Toxicology In vitro 22: $1726-1733$.

Wictome M, Michelangeli F, Lee AG, East JM. (1992). The inhibitors thapsigargin and 2,5-di(tert-butyl)-1,4-benzohydroquinone favour the E2 form of the $\mathrm{Ca}^{2+}, \mathrm{Mg}\left({ }^{2+}\right)$-ATPase. FEBS Lett 304: 109-13.

Williams SB, Cusco JA, Roddy MA, Johnstone MT, Creager MA. (1996). Impaired nitric oxide-mediated vasodilation in patients with non-insulin-dependent diabetes mellitus. J Am Coll Cardiol 27: 567-74.

Winkel-Shirley B. (2001). Flavonoid biosynthesis: a colorful model for genetics, biochemistry, cell biology and biotechnology. Plant Physiol 126: 485-493.

Yang CS, Wang X. (2010). Green tea and cancer prevention. Nutr Cancer 62: 931-937. Review.

Ying J, Sharov V, Xu S, Jiang B, Gerrity R, Schöneich C, Cohen RA. (2008). Cysteine-674 oxidation and degradation of sarcoplasmic reticulum $\mathrm{Ca}\left({ }^{2+}\right)$ ATPase in diabetic pig aorta. Free Radic Biol Med 45: 756-62.

Zheng J, Lee HC, Bin Sattar MM, Huang Y, Bian JS. (2011). Cardioprotective effects of epigallocatechin-3-gallate against doxorubicin-induced cardiomyocyte injury. Eur JPharmacol 652: 82-88. 